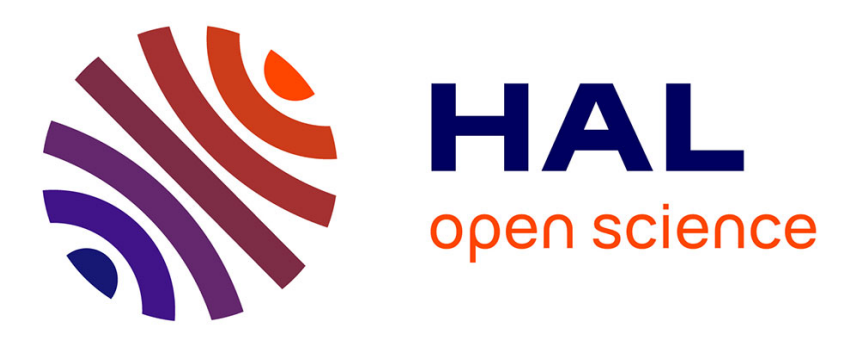

\title{
Influence of orthogonal prestrain on the viscoelastic behaviour of highly-filled elastomers
}

\author{
Aurélie Azoug, Anders Thorin, Robert Nevière, Rachel-Marie \\ Pradeilles-Duval, Andrei Constantinescu
}

\section{To cite this version:}

Aurélie Azoug, Anders Thorin, Robert Nevière, Rachel-Marie Pradeilles-Duval, Andrei Constantinescu. Influence of orthogonal prestrain on the viscoelastic behaviour of highly-filled elastomers. Polymer Testing, 2013, 32 (2), pp.375-384. 10.1016/j.polymertesting.2012.12.005 . hal-01402496

\author{
HAL Id: hal-01402496 \\ https://hal.science/hal-01402496
}

Submitted on 24 Nov 2016

HAL is a multi-disciplinary open access archive for the deposit and dissemination of scientific research documents, whether they are published or not. The documents may come from teaching and research institutions in France or abroad, or from public or private research centers.
L'archive ouverte pluridisciplinaire HAL, est destinée au dépôt et à la diffusion de documents scientifiques de niveau recherche, publiés ou non, émanant des établissements d'enseignement et de recherche français ou étrangers, des laboratoires publics ou privés. 
Test method

\title{
Influence of orthogonal prestrain on the viscoelastic behaviour of highly-filled elastomers
}

\author{
Aurélie Azoug ${ }^{\mathrm{a}, *}$, Anders Thorin ${ }^{\mathrm{a}}$, Robert Nevière ${ }^{\mathrm{b}}$, \\ Rachel-Marie Pradeilles-Duval ${ }^{\mathrm{a}}$, Andrei Constantinescu ${ }^{\mathrm{a}}$ \\ a Laboratoire de Mécanique des Solides, CNRS UMR 7649, Ecole Polytechnique, Palaiseau Cedex, France \\ ${ }^{\mathrm{b}}$ SNPE Matériaux Energétiques, Centre de Recherches du Bouchet, 9 Rue Lavoisier, 91710 Vert-le-Petit, France
}

\begin{abstract}
Highly filled elastomers exhibit a complex nonlinear mechanical behaviour that is difficult to characterize experimentally. This paper presents a Dynamic Mechanical Analysis (DMA) method coupled with orthogonal prestrains, applied in two distinct steps. A localization operator between measurements at the arms of a cross shaped specimen and the stress and strain fields at its center was determined using elastic small strain finite element computations. The operator makes estimating the storage and loss moduli at the center of the specimen possible. A mathematical model is then fitted to the moduli values. These results are compared to DMA measurements of highly filled elastomers under uniaxial prestrain. Although the storage and loss moduli increase with the prestrain under both loadings, the nonlinear behaviour is quantitatively modified by adding an orthogonal prestrain. In addition, the modification of the behaviour under a horizontal prestrain is cancelled out by an increase of the vertical prestrain, which may be explained by fillers aligning in the direction of the prestrain.
\end{abstract}

Keywords: Highly-filled elastomer Solid propellant, Dynamic Mechanical Analysis Prestrain, Fillers alignment

\section{Introduction}

Solid propellants are highly filled elastomers used for propulsion of rockets and launchers. Highly filled elasto mers have a filler volume fraction of up to $80 \%$. As a consequence, only a small quantity of binder holds the particles together and assures the viscoelastic properties of the composite up to the large strains. Due to the high filler fraction, the material exhibits highly nonlinear mechanical behaviour, and predictive material models are needed in order to design the structure of the motors.

The modelling of the nonlinear viscoelastic mechanical behaviour exhibited by these highly filled materials is a current research challenge. The offered models are either (i) phenomenological [ 1 5], which lead to imprecise pre dictions of the material behaviour under complex loadings or extreme environmental conditions (temperature), or (ii) based on homogenization theories [6 10] and extra polated to high filler ratios where their assumptions are not precisely verified. The difficulty of determining models based on the physics of the material (non phenomenolog ical models) arises from the complexity of the microscopic structure and the induced local deformation mechanisms. An additional obstacle arises from the involved binder filler and filler filler interactions. Measuring the mechanical behaviour of the material under complex loading opens doors to a better understanding of local deformation mechanisms.

The viscoelastic behaviour under prestrain has been previously studied by superimposing a tensile prestrain with small strain oscillations on unfilled rubber in tensile mode [11,12], on carbon black and silica filled elastomers 
in tensile and shear mode [13-20], and on highly-filled elastomers in torsion mode [21] and uniaxial tensile mode [22].

Suphadon et al. [23,24] compared the loss modulus of unfilled and filled rubbers subject to a uniaxial tensile prestrain and either tension or torsion strain oscillations. They showed that the loss modulus becomes anisotropic at large prestrains.

The objective of this paper is to present a series of measurements of the viscoelastic behaviour of propellant under two orthogonal prestrains. The prestrained Dynamic Mechanical Analysis (DMA) measurements are performed on cross-shaped specimens with two constant additional orthogonal prestrains. Using a simple formula, we estimate the stress and strain fields in the center of the specimen and establish a nonlinear model for the viscoelastic behaviour of the composite under orthogonal prestrains.

\section{Materials}

The solid propellants studied here are highly-filled elastomers characterized by a filler fraction larger than $86 \%$ wt. The fillers are ammonium perchlorate and aluminum particles. The binder is based on hydroxy-terminated polybutadiene (HTPB) prepolymer (the functionality of the polymer is slightly higher than 2) cured with a methylene diicyclohexyl isocyanate (MDCI). The properties of the system depend on the cross-link density of the binder, which can be characterized by the $\mathrm{NCO} / \mathrm{OH}$ ratio. The latter is the ratio of the molar quantities of MDCI and HTPB prepolymer introduced during manufacture. If the $\mathrm{NCO} / \mathrm{OH}$ ratio is lower than 1 , the system does not contain enough cross-linking agents to create a complete network and, consequently, part of the HTPB molecules remains unlinked to the network.

A plasticizer, dioctyl azelate (DOZ) molecules, is introduced in the mixture. Filler-binder bonding agents (FBBA) can also be added. These molecules react chemically with the fillers and with the polymer chains, creating links between the surface of the fillers and the polymer molecules. These chemical links have an influence on the mechanical behaviour of the material.

The materials are thermally cured at $50{ }^{\circ} \mathrm{C}$ for two weeks.

In this study, we compare five solid propellants, differing in filler fraction, $\mathrm{NCO} / \mathrm{OH}$ ratio, plasticizer content, and presence or absence of FBBA. Their composition is detailed in Table 1.

Table 1

Composition of the studied propellants. FBBA: presence $(x)$ or absence $(-)$.

\begin{tabular}{lllll}
\hline & $\begin{array}{l}\text { Filler fraction } \\
(\% w t)\end{array}$ & $\begin{array}{l}\text { NCO/OH } \\
\text { ratio (-) }\end{array}$ & $\begin{array}{l}\text { Plasticizer } \\
\text { (\%wt of the binder) }\end{array}$ & FBBA (-) \\
\hline A & 86 & 0.80 & 10.0 & $\times$ \\
B & 88 & 0.80 & 22.5 & $\times$ \\
C & 90 & 0.80 & 30.0 & - \\
D & 88 & 0.88 & 25.0 & - \\
E & 90 & 0.95 & 20.0 & - \\
\hline
\end{tabular}

\section{Experimental procedure}

\subsection{Specimen and loading}

Cross-shaped specimens were cut from thick plates of each material (Fig. 1). The arms of the cross were connected with large radii in order to limit stress concentrations due to the orthogonal strain field. Overall dimensions of the specimens are $50 \mathrm{~mm} \times 45 \mathrm{~mm} \times 5 \mathrm{~mm}$.

In order to apply the orthogonal prestrain, a particular set up was constructed out of grips and calibrated beams (Fig. 1).

The loading of the specimen is performed in three steps (Fig. 2):

Step 1: A horizontal prestrain is applied using a standard tensile machine. $e_{1}=0 \%, 2 \%, 5 \%$, or $7.5 \%$.

Step 2: The displacement is maintained with rigid beams. Grips are stuck to the surfaces of the specimen facing the vertical direction. The stresses within the specimen are then relaxed for at least $48 \mathrm{~h}$ and are considered to have reached a stable value.

Step 3: The set up is finally placed into the DMA apparatus (Metravib Viscoanalyseur VA3000) and is loaded on the vertical axis with a prestrain $e_{2, i}$ ranging between 0.01 and $7.5 \%$, added to strain oscillations with a frequency $f=5 \mathrm{~Hz}$ and an amplitude $e_{a}=0.01 \%$. In the end, the total strain in the direction of the axes according to time is

$e_{1}(t)=e_{1}$ in the horizontal axis

$e_{2}(t)=e_{2}+e_{a} \sin (2 \pi f t)$ in the vertical axis

The tests are performed at room temperature. A schematic representation of the imposed loading is proposed in Fig. 2d.

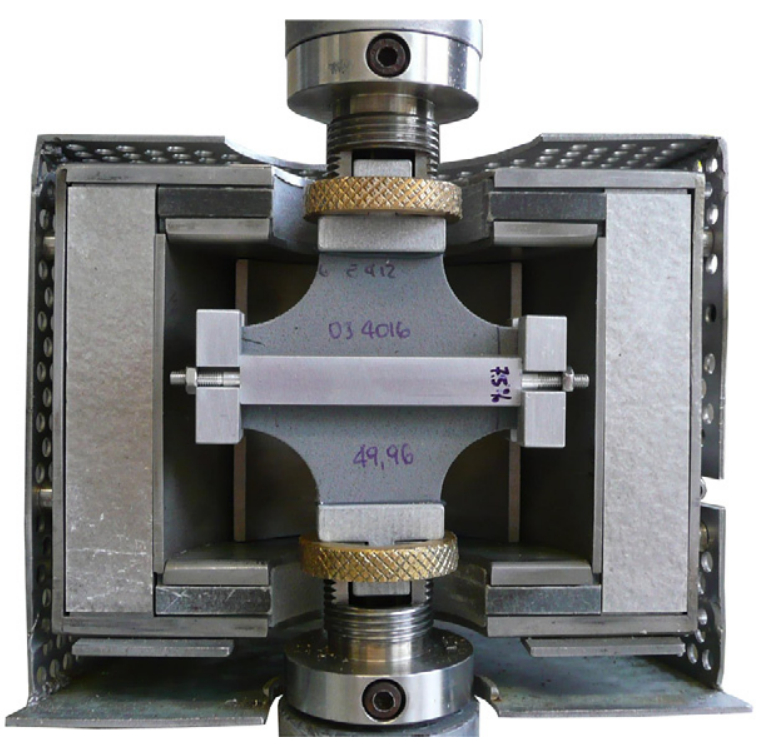

Fig. 1. The orthogonally prestrained set up in the DMA testing device. 


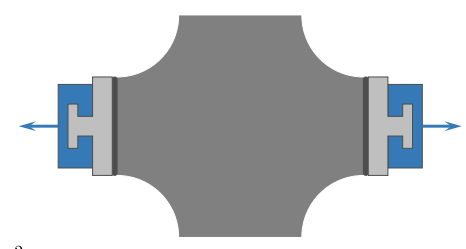
$\stackrel{2}{\longrightarrow}$

a Step 1

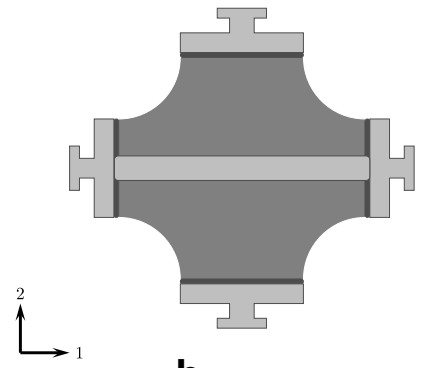

b Step 2
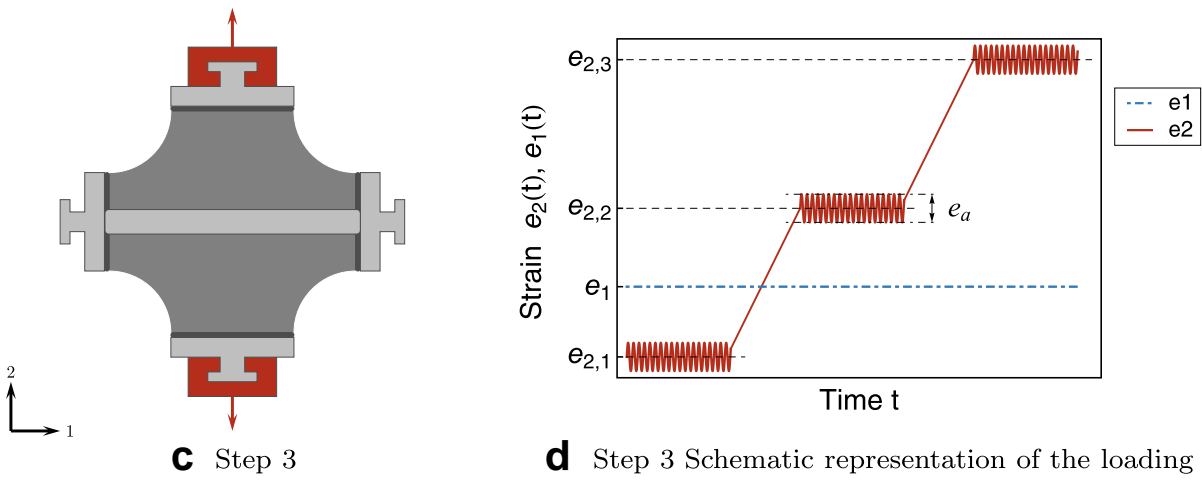

Fig. 2. Steps of the DMA test under orthogonal prestrain.

In the final step, the DMA apparatus computes the loss factor, $\tan \delta$, and the apparent stiffness of the specimen, $K$, defined as the ratio between the vertical measured forces and the imposed displacement. Consequently, $K$ depends on the shape and dimensions of the cross-shaped specimen. The strain field, $\varepsilon$, is represented in Fig. 3 under the assumption of small strain and an imposed displacement of $1 \mathrm{~mm}$ in both orthogonal directions. Fig. 3 illustrates the fact that the strain and stress fields are not homogeneous in the specimen and, therefore, that $K$ is not an intrinsic material property.

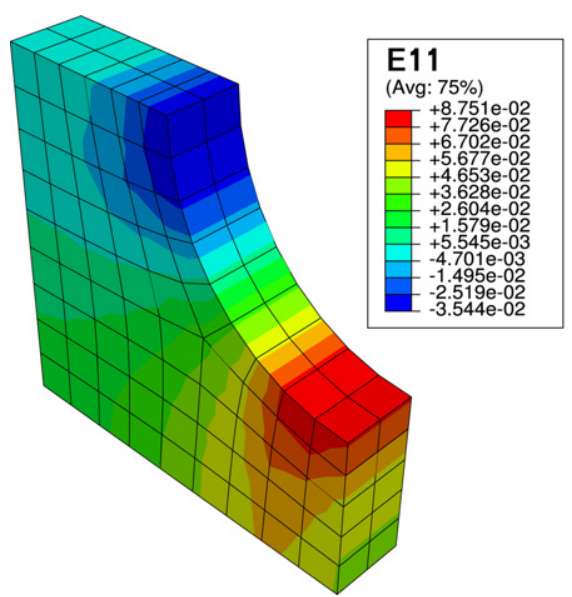

The key point is to estimate the local mechanical fields in the center of the cross-shaped specimen. To achieve this, one could perform full field strain measurements on the surface of the specimen. However, due to the transient character of the measured fields, the experimental setup would be complex and would need additional interpretation.

The method developed here is to determine the local strain and stress fields in the center of the cross-shaped specimen from the measured forces and imposed displacements at the grips using a localization operator.

Fig. 3. The heterogeneity of the strain field (horizontal and vertical components, i.e. $\varepsilon_{11}$ and $\varepsilon_{22}$ ) under the assumption of small strains and an imposed displacement of $1 \mathrm{~mm}$ in both orthogonal directions. 
The localization operator was computed using a finite element modelling that is presented in the next section.

\subsection{The localization operator}

The localization operator is represented by two matrices that permit an estimation of the strain and stress fields at the center of the specimen from the measured forces and the imposed displacements at the grips. This is done under a series of simplifying assumptions. The material is considered to be under small strains and to exhibit linear viscoelastic behaviour. Therefore, one can further accept that the phase shift angle $\delta$ is constant over the specimen and that the local strain and stress fields are obtained using an elastic computation taking advantage of the superposition principle as:

$\widehat{\sigma}=L_{\sigma} \sigma$

$\widehat{\varepsilon}=L_{\varepsilon} e$,

where $\widehat{\sigma}$ and $\widehat{\varepsilon}$ denote the stress and strain tensors at the center of the specimen (Fig. 4), and $\sigma$ and $e$ denote the stress and strain defined hereafter. Numerical computations showed that only the stress components along the horizontal and vertical coordinate axes are significant. A similar observation holds for the strains with the exception of the through-thickness strain component, which is related to the horizontal and vertical components by the incompressibility constraint of the material. As a consequence, we only consider the stress and strain components along the horizontal and vertical axes, which are denoted $\widehat{\sigma}_{i}$ and $\widehat{\varepsilon}_{i}$ for $i=1,2$. The macroscopic values $\sigma=\left(\sigma_{1}, \sigma_{2}\right)$ and $e=\left(e_{1}, e_{2}\right)$ are computed with the formulae:

$\sigma_{i}=\frac{F_{i}}{S_{i}}$

$e_{i}=\frac{U_{i}}{D_{i}}$

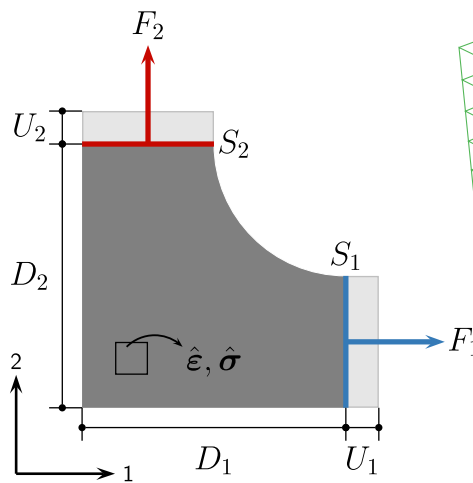

a Notations for the macrosopic and local values

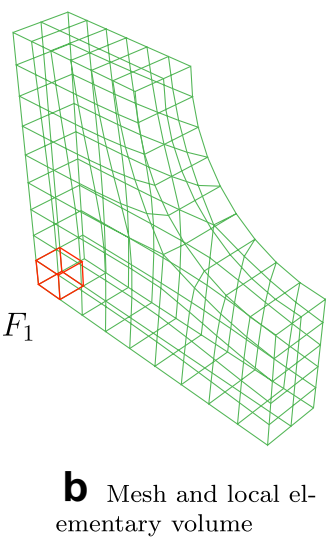

Fig. 4. The characteristics defining the macroscopic values $\sigma$ and $e$ : experimental forces $F$, surfaces $S$, displacements $U$, and dimensions $D$. from forces $F_{i}$, surfaces $S_{i}$, displacements $U_{i}$, and dimensions $D_{i}$, for $i=1,2$ (see Fig. 4 a).

The horizontal stress $\sigma_{1}$ was estimated from numerical computations with imposed displacements. As a consequence, the evolution of this value outside the linear domain is unknown.

The main assumptions leading to the localization operator are: (a) small strains, (b) homogeneous strains and stresses in the local elementary volume, and (c) the similarity of the spatial distributions of the fields in linear elasticity and viscoelasticity. To assess the accuracy of the results, the validity of the assumptions (a) and (b) was verified by several computational tests, for a quasiincompressible (Poisson's ratio $\nu=0.48$ ) model material with unit Young modulus $(E=1 \mathrm{~Pa})$.

The small strain assumption was numerically tested for strains $e$ between 0 and $10 \%$. The range of validity of the approximation of stress and strain fields in the center using the localization matrix is obtained for maximal strains in the horizontal and vertical directions of $e_{1}^{\max }=4.0 \%$ and $e_{2}^{\max }=4.5 \%$, respectively. For these strains, the error between the stress components computed in large strains and small strains is of $5.9 \%$ in direction 1 and of $5.1 \%$ in direction 2.

Because the experiments imposing a horizontal prestrain $e_{1}=7.5 \%$ lie outside of the validated range, they were not interpreted using this technique. Nevertheless, if either $e_{1}$ or $e_{2}$ is small and the other is reasonably greater than $5 \%$, the small strains assumption is still relevant. As an example, for $e_{1}=5.0 \%$ and $e_{2}=2.0 \%$, the assumption of small strains introduces a $0.8 \%$ error on the stress components.

The assumption of a homogeneous elementary cell in the center of the specimen is also valid for some given loadings. The stress field in one element is compared to the values obtained in the neighboring elements, again at $e_{1}=4.0 \%$ and $e_{2}=4.5 \%$ (the obtained values of the stress components are displayed in Fig. 5). Similar results can be obtained for the strain components. We can conclude that the relative heterogeneity is up to $6.3 \%$ in stress and $16 \%$ in strain for $e_{1}=4.0 \%$ and $e_{2}=4.5 \%$.

Finally, the last assumption, namely the similarity of the spatial distributions of the fields in linear elasticity and viscoelasticity, is discussed. Although the influence of the loading history on the distribution of strain and stress fields in the specimen was not quantified, several observations led to this assumption. During step 2 of the experimental procedure, the stresses in the specimen were relaxed for $48 \mathrm{~h}$ after applying the horizontal prestrain. The stress state reached at the end of step 2 is considered fully relaxed compared to the timescale of the DMA test [25]. At step 3 , the duration between the application of the vertical prestrain and the application of the DMA cyclic loading was a few minutes, which has been checked to allow for a constant measurement value [25]. Consequently, the simulation of the field distributions in a linear elastic specimen is assumed to be a close approximation of those same field distributions in a linear viscoelastic material. In addition, the phase shift $\delta$ between the stress and strain fields in the center of the specimen, $\widehat{\sigma}$ and $\widehat{\varepsilon}$, is assumed to be identical to the phase shift between the macroscopic stress and strain values, $\sigma$ and $e$. 

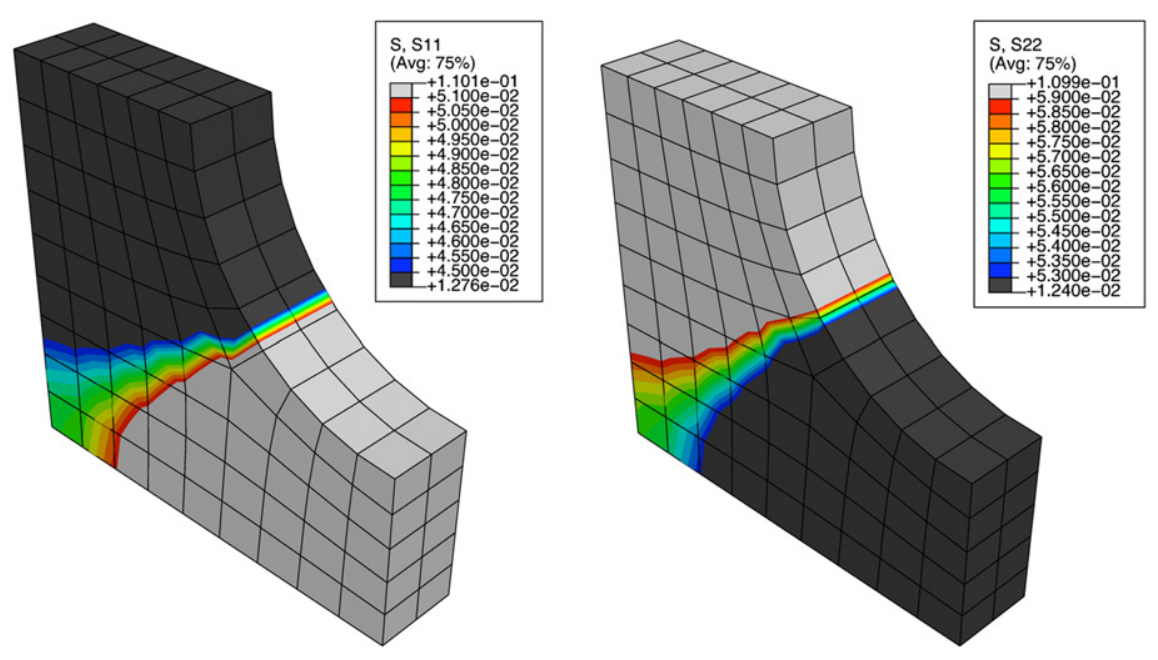

Fig. 5. Heterogeneous stress components $\sigma_{11}$ and $\sigma_{22}$ in the cross-shaped specimen, loading $e_{1}=4.0 \%, e_{2}=4.5 \%$.

\section{The model}

The magnitude of the complex modulus $\left\|E^{*}\right\|$ is determined from the ratio of the amplitude of the sinusoidal stress $\widehat{\sigma}_{2}$ and strain $\widehat{\varepsilon}_{2}$. We recall that the loss factor is assumed to be constant over the whole geometry. The storage and loss moduli, $E^{\prime}$ and $E^{\prime \prime}$, are deduced from $\left\|E^{*}\right\|$ and $\tan \delta$ using Equation (7).

$\left\|E^{*}\right\|=\sqrt{E^{\prime 2}+E^{\prime \prime 2}}, \tan \delta=\frac{E^{\prime \prime}}{E^{\prime}}$

The storage modulus $E^{\prime}$ quantifies the elastic part of the behaviour, while the loss modulus $E^{\prime \prime}$ corresponds to the quantity of heat dissipated by friction between polymer chains during a strain cycle [26].

For both the storage modulus $E^{\prime}$ and the loss modulus $E^{\prime \prime}$, we define a unique model, Equation (8), which approximates the dependency of both curves on the prestrain $e_{2}$.

$X\left(e_{2}\right)= \begin{cases}\alpha+\beta \log \left(e_{2}\right) & \text { if } e_{2}<e_{t}, \\ \alpha+\beta \log \left(e_{2}\right)+\gamma\left[\log \left(\frac{e_{2}}{e_{t}}\right)\right]^{\zeta} & \text { otherwise, }\end{cases}$

where $X$ stands for $E^{\prime}$ or $E^{\prime \prime}$ and $\alpha, \beta, e_{t}, \gamma$, and $\zeta$ are real numbers considered as material parameters. Each modulus, $E^{\prime}$ or $E^{\prime \prime}$, is thus defined by its set of material parameters. A similar model with a piece-wise loglinear function and a uniaxial prestrain is described in [22,27].

This mathematical model is optimized to the experimental results using a least-squares optimization algorithm in Mathematica ${ }^{\circledR}$. More precisely, $\alpha$ and $\beta$ are first determined from at least four measurements at low prestrain. The optimal values of least square distance with respect to $e_{t}, \gamma$, and $\zeta$ are then determined from the second case of Equation (8). Confidence intervals at a $95 \%$ level are computed numerically for each parameter in Mathematica ${ }^{\circledR}$.

The viscoelastic behaviour measured by DMA is characterized by three quantities, as described in Fig. 6 . The plateau value $P$ is chosen to be the model value at $e_{2}=e_{p}$, see Equation (9). The nonlinearity threshold $T$ is directly given by $e_{t}$. The nonlinearity slope $S$ is defined as the slope of the logarithmic asymptote of the curve (Fig. 6) and is determined from constants $e_{t}, \beta, \gamma$, and $\zeta$ according to Equation (10).

$P=\alpha+\beta \log \left(e_{p}\right)$,

$S=\frac{1}{N} \sum_{i=1}^{N}\left(\beta+\gamma \zeta\left[\log \left(\frac{e_{t}+e_{2, i}}{e_{t}}\right)\right]^{\zeta-1}\right)$,

where $e_{p}=0.01 \%, e_{2, i} \in[3 \%, 6 \%]$, and $N=16$.

In the following sections, the superscripts $(\cdot)^{\prime}$ and $(\cdot)^{\prime \prime}$ are added to the parameters of the model to denote the application for the storage and loss modulus, respectively. Confidence intervals at a 95\% level for $P, T$, and $S$ are computed from the ones of the model parameter $\alpha, \beta, e_{t}, \gamma$, and $\zeta$.

\section{Results and discussion}

The experimental results are presented and discussed using the model defined in Equation (8). Only the

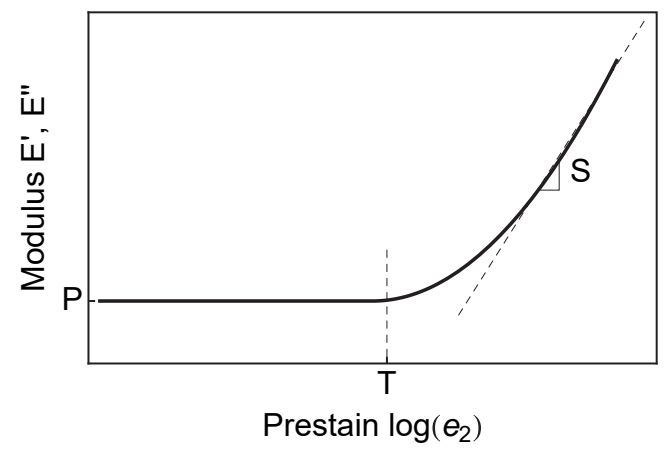

Fig. 6. Schematic representation of the quantification of the viscoelastic behaviour. 
experiments with a horizontal prestrain $e_{1}$ of 0,2 , and $5 \%$ are used, as the results at $7.5 \%$ lie outside the limit of accuracy of the localization formulae. However, using the scores obtained at high vertical prestrains $e_{2}$ stabilized the identification of the model, especially the threshold.

\subsection{Comparison of the behaviour under uniaxial and orthogonal prestrain}

Before comparing the results, it is important to note that the two experiments are not equivalent. In uniaxial DMA, the horizontal stress $\sigma_{1}$ is zero, whereas in the orthogonally prestrained DMA, the horizontal macroscopic strain $e_{1}$ is fixed at zero. The material behaviour under orthogonal prestrain is qualitatively similar to that observed under uniaxial prestrain. The model defined in (8) was used for identification in both experiments.

Let us start with an inspection of the parameters of the model in uniaxial and orthogonal prestrain as presented in Fig. 6.

Fig. 7 displays the value of the parameter $P$ characterizing the height of the plateau for each material composition. The displayed table represents the relative changes of the parameter $P$ for the orthogonal measurement with respect to the uniaxial measurement. More precisely, the first and second rows of the table are defined as:

$\frac{P_{\text {orthogonal }}^{\prime}-P_{\text {uniaxial }}^{\prime}}{P_{\text {uniaxial }}^{\prime}}$ and $\frac{P_{\text {orthogonal }}^{\prime \prime}-P_{\text {uniaxial }}^{\prime \prime}}{P_{\text {uniaxial }}^{\prime \prime}}$.

Materials $\mathrm{C}$ and $\mathrm{E}$, which are more heavily loaded compared to the others, exhibit particular behaviour. Material E manifests higher plateau values $P^{\prime}$ than the ones of the other materials, whether in uniaxial or orthogonal prestrained DMA. In addition to being heavily loaded, this material is highly cross-linked, which leads to a dense network. The high storage modulus quantifies the large elastic part of the behaviour.

Although material C is heavily loaded, it is also plasticised and less cross-linked. As a consequence, the plateau values are low compared to material E. However, one can note that the relative increase in $P$ is significantly larger for material $C$. The nonlinear behaviour under orthogonal

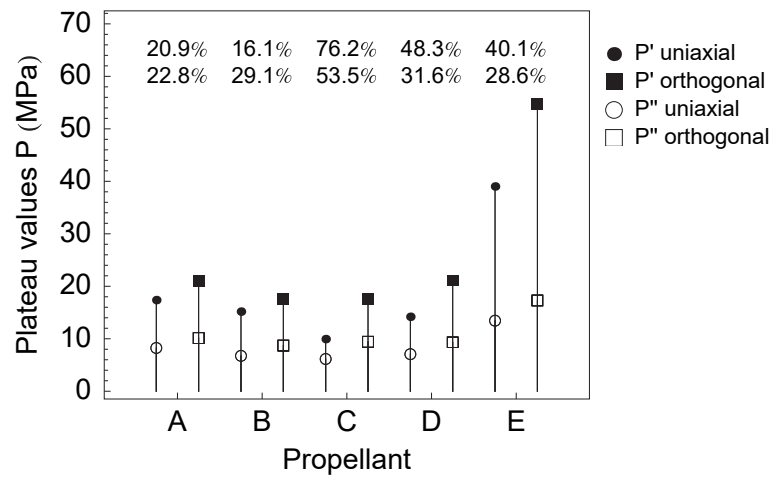

Fig. 7. Comparison of the plateau values $P$ of DMA tests under uniaxial and orthogonal prestrain $\left(e_{1}=0\right)$.

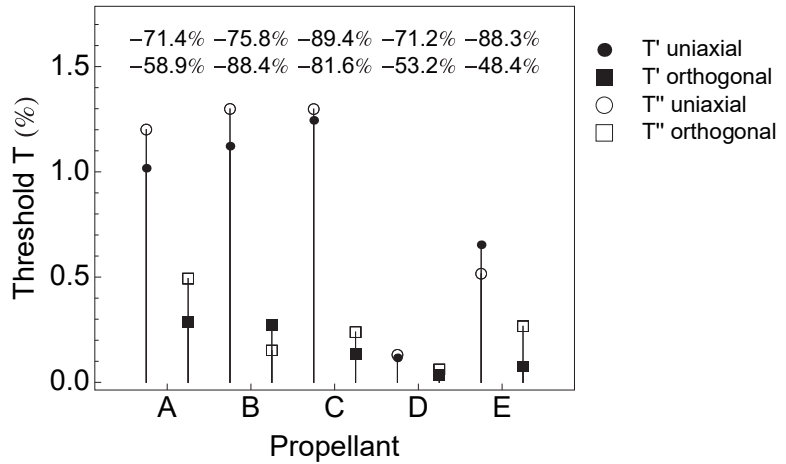

Fig. 8. Comparison of the nonlinearity thresholds $T$ of DMA tests under uniaxial and orthogonal prestrain $\left(e_{1}=0\right)$.

prestrain probably originates from the combination of a high filler fraction and a low cross-link density network.

The observed differences between heavily loaded materials and the others could be explained by the filler rearrangement mechanism proposed in [3] or by the formation of stress concentration bands as shown by [28] and observed in [29].

The nonlinearity of the material as a function of the vertical prestrain $e_{2}$ is also modified by the presence of a horizontal prestrain $e_{1}$ (Figs. 8 and 9). The nonlinearity thresholds $T^{\prime}$ and $T^{\prime \prime}$ decrease by at least $71.2 \%$ and $48.4 \%$, respectively (Fig. 8).

The identification results obtained through different runs of the algorithm showed a small confidence interval for the plateau $P$ and the slope $S$, and a large one for the threshold $T$. This is to be expected as a small change in $S$ induces a larger change in $T$.

The elongation at failure of a propellant is divided by two in equibiaxial loading with respect to uniaxial loading [29]. Therefore, a decrease of the nonlinearity threshold $T$ measured under orthogonal prestrain is expected when compared to measurements under uniaxial prestrain.

It appears that the nonlinearity, as expressed by the slope $S$, is accentuated by the presence of an orthogonal prestrain $e_{1}$ (see Fig. 9). However, a larger number of experiments have to be performed to assess the physical interpretation.

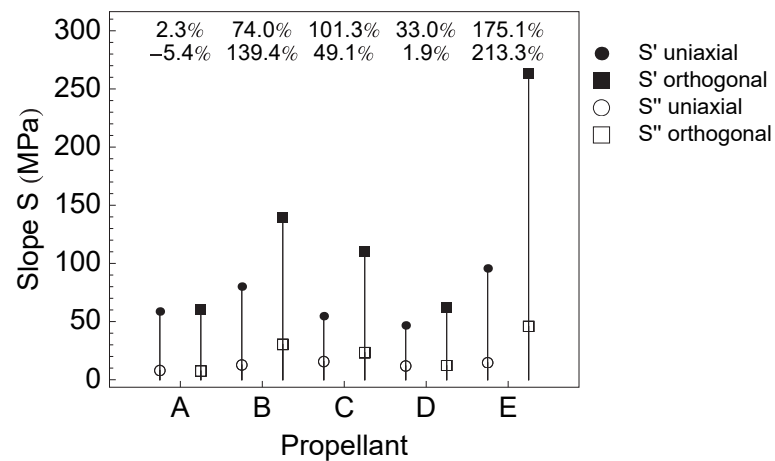

Fig. 9. Comparison of the nonlinearity slopes $S$ of DMA tests under uniaxial and orthogonal prestrain $\left(e_{1}=0\right)$. 

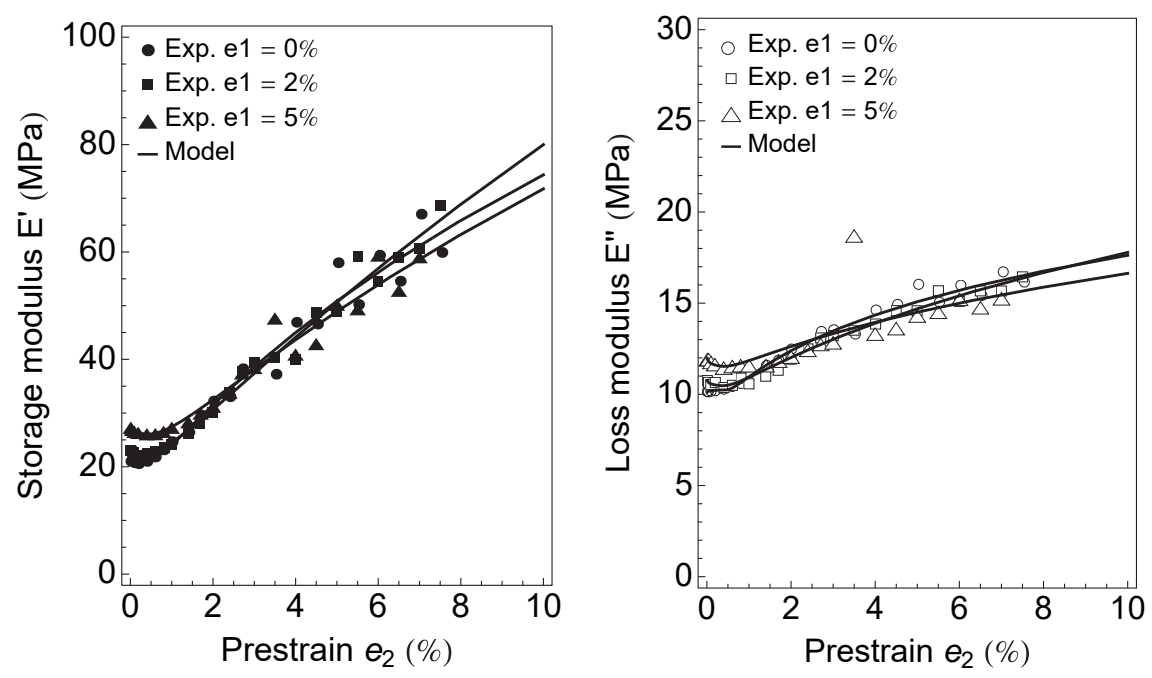

Fig. 10. Storage and loss modulus of propellant A, measured under orthogonal prestrain, $\left(f=5 \mathrm{~Hz}, e_{a}=0.01 \%\right)$.

\subsection{Nonlinear domain}

The orthogonal experiments were interpreted using the proposed model for uniaxial DMA experiments (Equation (8)) producing a reasonable correlation (see Figs. 11-15). The plotted $95 \%$ confidence intervals of the model are small and express a good fit of the model with experiments. An exception is encountered around the threshold values between the plateau and the slope, which was already found to be a weak point in the model. One can also note large confidence intervals for large vertical prestrains $e_{2}$ (see Fig. 15), but this phenomenon could probably be reduced by using a larger number of measurements.

Fig. 10 displays the storage and loss moduli, $E^{\prime}$ and $E^{\prime \prime}$, as functions of $e_{2}$. In order to highlight the model parameters $P, T$, and $S$, the next figures (Figs. 11-15) are plotted in loglinear coordinates.
One can note that the curves are largely spread in the region of the linear plateau, but they cluster once the threshold is reached. Starting from a level of prestrain $\left(e_{2}=e_{t}\right)$ that depends on the composition, the curves for the different values of prestrain $e_{1}$ merge and present similar behaviour. This observation indicates that the vertical prestrain $e_{2}$ compensates the effect of the horizontal prestrain $e_{1}$. Thus, if one supposes that the prestrain leads to a rearrangement mechanism of the anisotropic microstructure, then the previous observation implies that the rearrangement is cancelled out by a prestrain in an orthogonal direction.

The study of the model parameters $P, T$, and $S$, as functions of $e_{1}$ confirms these visual observations. The influence of the prestrain $e_{1}$ on the value of the horizontal plateau is the same for every material composition, see Table 2 . When $e_{1}$ increases from 0 to $5 \%$, the values of $P^{\prime}$ and $P^{\prime \prime}$ increase on
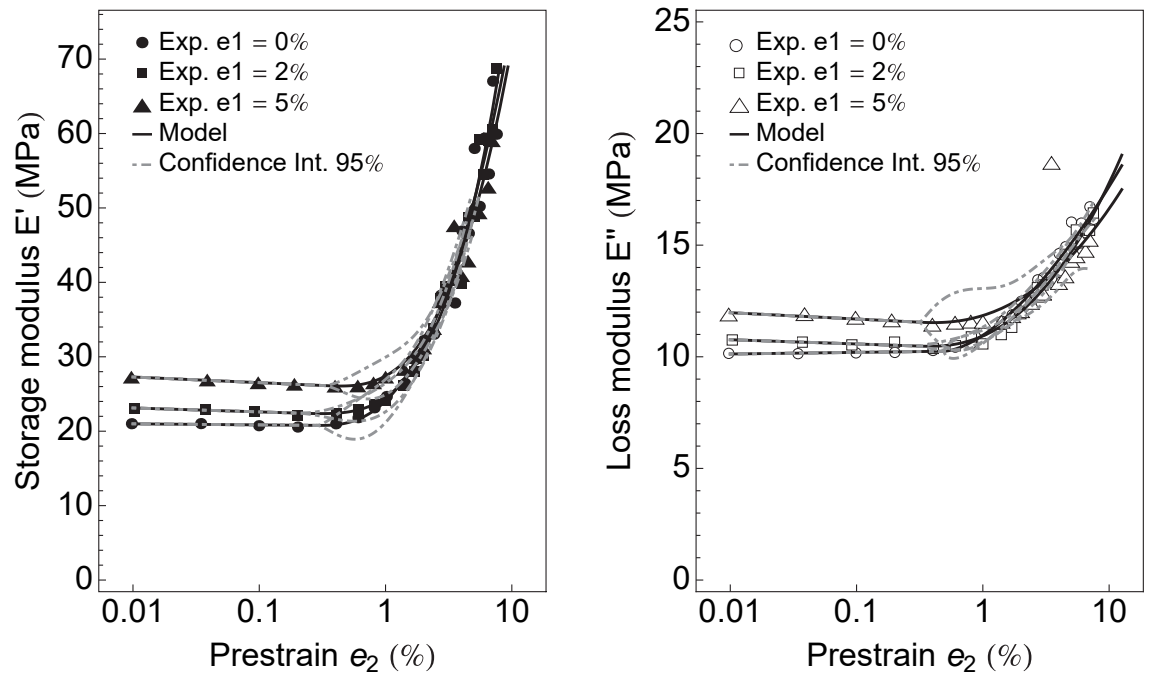

Fig. 11. Storage and loss modulus of propellant A, measured under orthogonal prestrain, $\left(f=5 \mathrm{~Hz}, e_{a}=0.01 \%\right)$. 

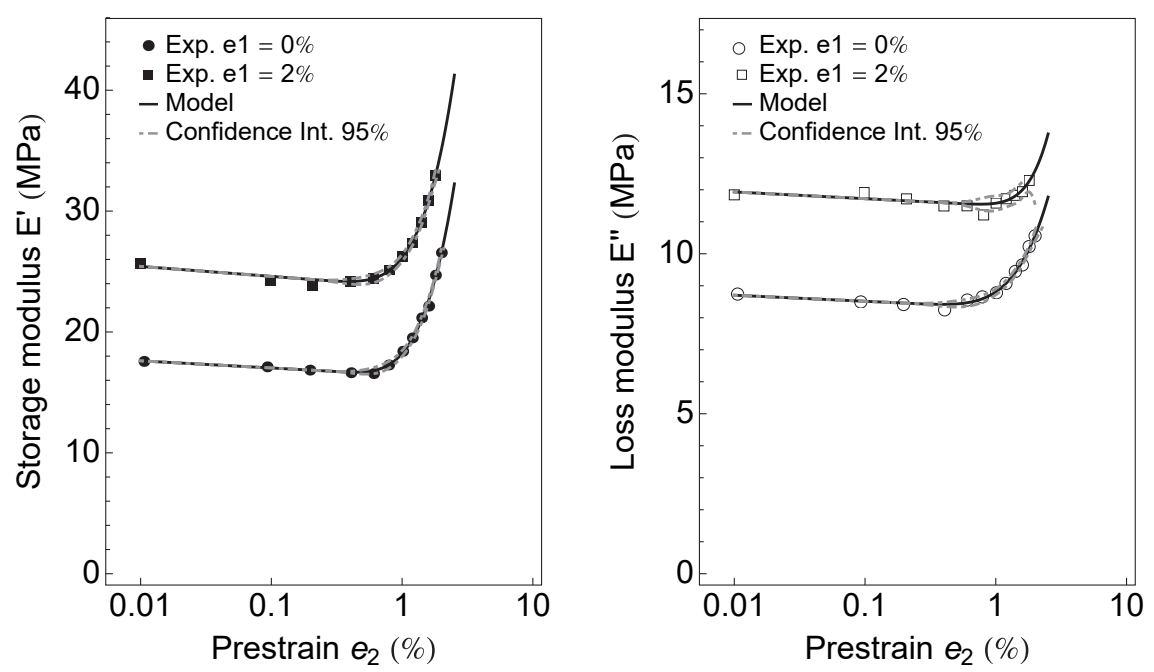

Fig. 12. Storage and loss modulus of propellant $\mathrm{B}$, measured under orthogonal prestrain, $\left(f=5 \mathrm{~Hz}, e_{a}=0.01 \%\right)$.
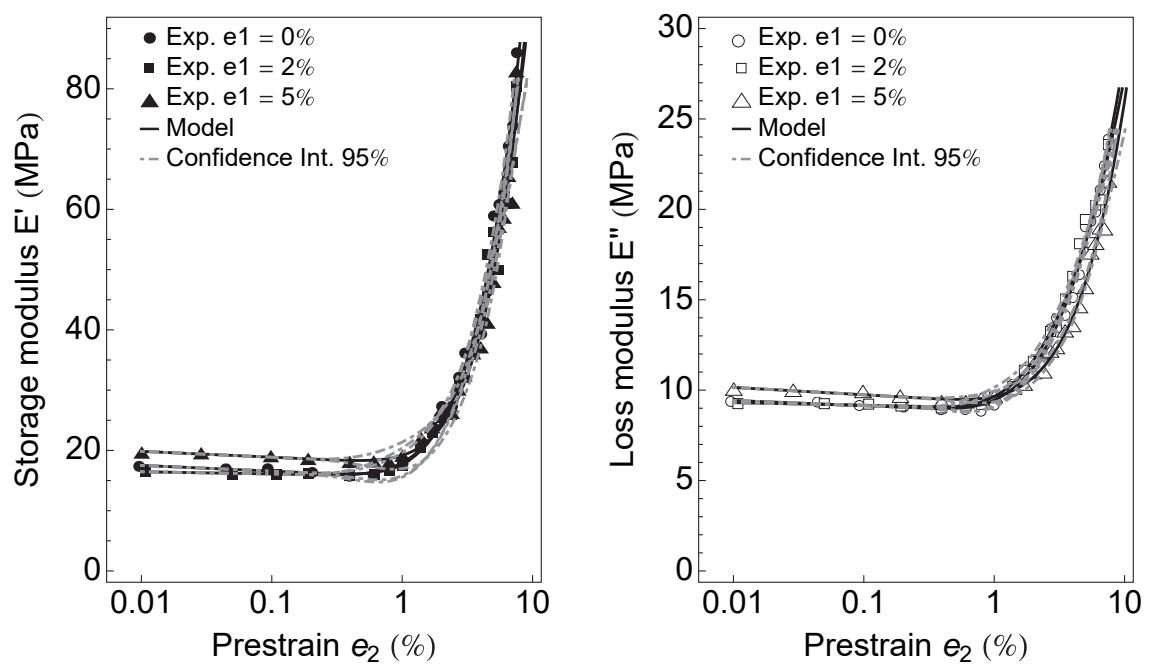

Fig. 13. Storage and loss modulus of propellant $\mathrm{C}$, measured under orthogonal prestrain, $\left(f=5 \mathrm{~Hz}, e_{a}=0.01 \%\right)$.
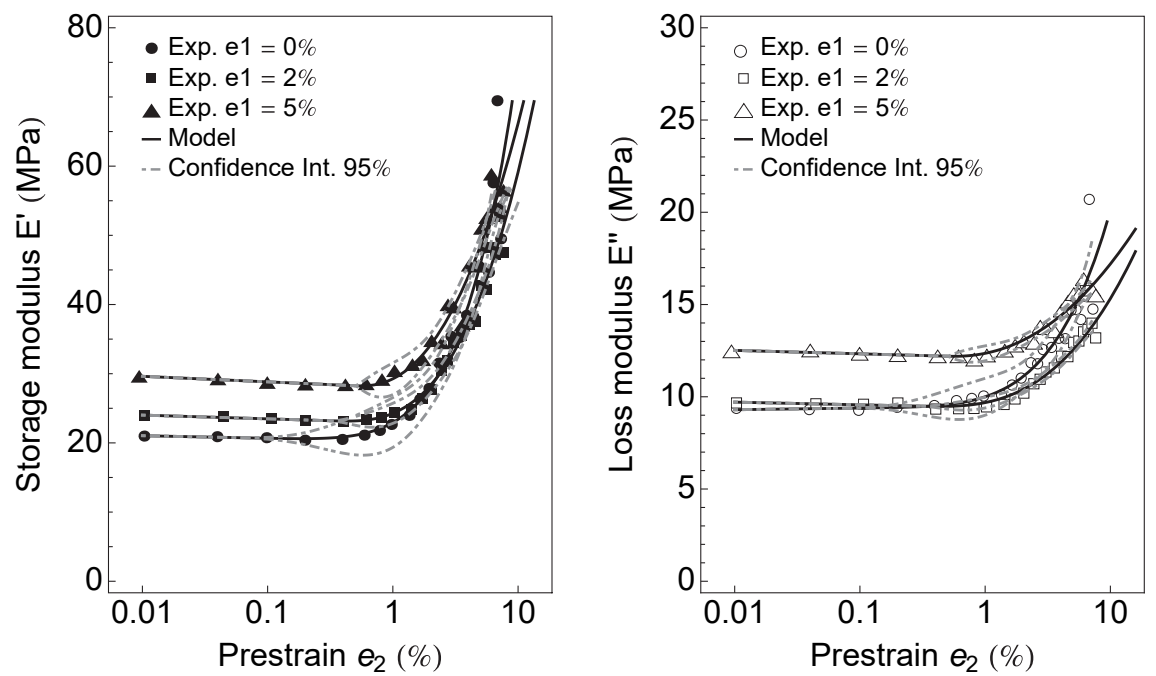

Fig. 14. Storage and loss modulus of propellant $\mathrm{D}$, measured under orthogonal prestrain, $\left(f=5 \mathrm{~Hz}, e_{a}=0.01 \%\right)$. 

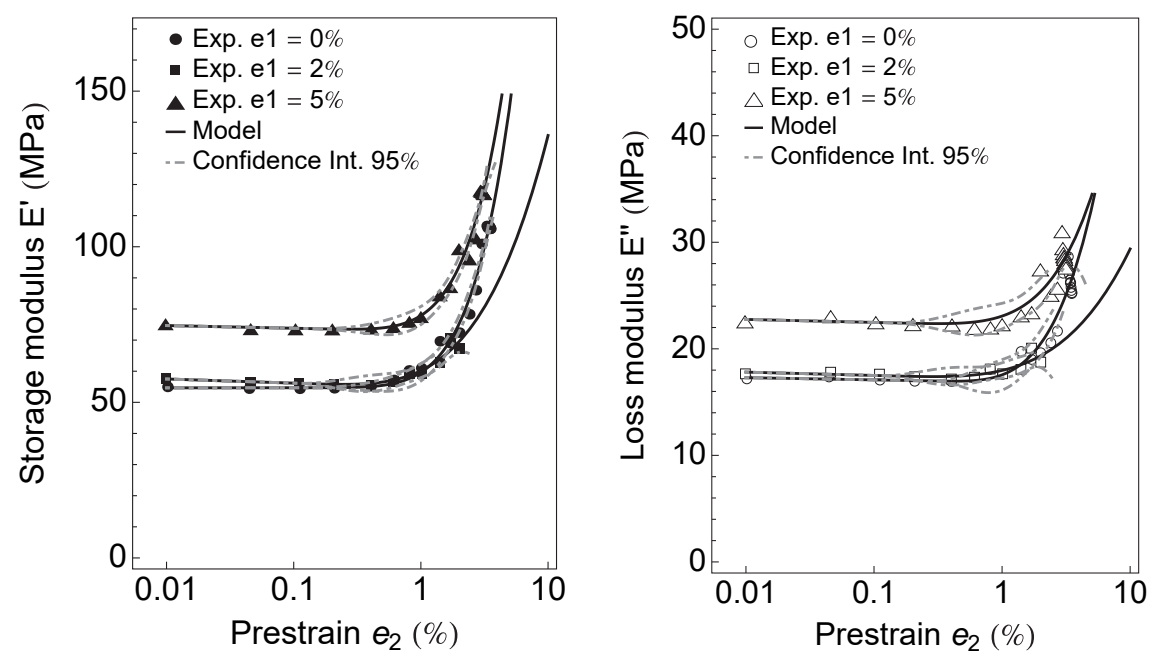

Fig. 15. Storage and loss modulus of propellant $\mathrm{E}$, measured under orthogonal prestrain, $\left(f=5 \mathrm{~Hz}, e_{a}=0.01 \%\right)$.

average by 33 and 26\%, respectively. This evolution is coherent with the effect of orthogonality observed in the previous paragraph.

Table 2 presents a peculiar value of the threshold when $e_{1}=2 \%$. This is a known defect of the proposed model [22]. The interpretation of these results is subject to much uncertainty. The uniaxial experiments showed that the uniaxial nonlinearity threshold is lower than $1.5 \%$ for every material. Moreover, the proposed model has different behaviour before and after the threshold. These two facts lead to a high variability in the model parameters for a prestrain $e_{1}$ close to the uniaxial nonlinearity threshold. The variability of the model parameters in the case of $e_{1}=2 \%$ can be recognized as a consequence of the perturbation of the behaviour around the uniaxial nonlinearity threshold.

Finally, increasing the prestrain $e_{1}$ decreases the slope of the nonlinearity as a function of $e_{2}$, see Table 2 . That means that the nonlinearity slope decreases as the prestrain varies from a uniaxial to an equibiaxial state. The slopes decrease between 2 and 50\%, except those of material B, which

Table 2

Parameters $P^{\prime}, P^{\prime \prime}, T^{\prime}, T^{\prime \prime}, S^{\prime}, S^{\prime \prime}$ for all studied materials and prestrains $e_{1}$ in orthogonal experiments.

\begin{tabular}{llllllrc}
\hline & $e_{1}(\%)$ & $P^{\prime}(\mathrm{MPa})$ & $P^{\prime \prime}(\mathrm{MPa})$ & $T^{\prime}(\%)$ & $T^{\prime \prime}(\%)$ & $S^{\prime}(\mathrm{MPa})$ & $S^{\prime \prime}(\mathrm{MPa})$ \\
\hline $\mathrm{A}$ & 0 & 21.0 & 10.1 & 0.29 & 0.49 & 60.0 & 7.5 \\
& 2 & 23.1 & 10.8 & 0.18 & 0.32 & 67.1 & 8.1 \\
& 5 & 27.3 & 12.0 & 0.32 & 0.32 & 54.1 & 5.7 \\
$\mathrm{~B}$ & 0 & 17.6 & 8.7 & 0.27 & 0.15 & 139.3 & 30.4 \\
& 2 & 25.4 & 11.9 & 0.16 & 0.32 & 157.8 & 54.2 \\
$\mathrm{C}$ & 0 & 17.5 & 9.4 & 0.13 & 0.24 & 110.0 & 23.3 \\
& 2 & 16.5 & 9.3 & 0.13 & 0.32 & 96.4 & 21.5 \\
& 5 & 19.8 & 10.2 & 0.10 & 0.15 & 98.6 & 19.6 \\
$\mathrm{D}$ & 0 & 21.0 & 9.3 & 0.03 & 0.06 & 62.1 & 12.0 \\
& 2 & 24.0 & 9.7 & 0.32 & 0.32 & 42.5 & 6.8 \\
& 5 & 29.6 & 12.5 & 0.50 & 0.50 & 45.0 & 5.8 \\
$\mathrm{E}$ & 0 & 54.7 & 17.3 & 0.08 & 0.27 & 263.2 & 45.8 \\
& 2 & 57.5 & 17.8 & 0.10 & 0.10 & 90.5 & 13.7 \\
& 5 & 74.7 & 22.8 & 0.08 & 0.10 & 274.3 & 32.2 \\
\hline
\end{tabular}

increase. However, adding measurements at prestrains $e_{2}$ higher than $2 \%$ would change the extrapolated value of the slopes for material B. The decrease of the slope explains the fact that the measurements are identical for large vertical prestrains $e_{2}$, despite the wide variation in thresholds.

\section{Conclusions and perspectives}

The material viscoelastic behaviour under orthogonal prestrain is complex and difficult to characterize experimentally. This study presents a method of conducting DMA experiments with orthogonal prestrain. The prestrain was applied in two orthogonal directions in two distinct steps separated by a resting period. The performed measurements provided the values of the apparent stiffness $K$ and the loss factor $\tan \delta$ at different prestrains in the vertical and horizontal directions.

In order to interpret the measurements and obtain some intrinsic material properties, a relationship between the mean strain and stress at the arms of the cross specimen and the local stress and strain fields in the center of the cross specimen was proposed. The relations were obtained under a series of assumptions, essentially small strains and linear material behaviour, using finite element computations. The limit of the approximations was clearly identified and did not restrain the validity of the present results. These relations allowed estimating the storage and loss modulus of the material from the measurements.

The measurements under an orthogonal prestrain were then identified using a mathematical model proposed for the results of uniaxial DMA. The model predictions of $E^{\prime}$ and $E^{\prime \prime}$ were close to the measured values and provided a small confidence interval with respect to the model parameters.

The material behaviour under orthogonal prestrain is qualitatively similar to that observed under uniaxial prestrain. The nonlinearity is highly modified by imposing a zero horizontal prestrain, which is different from the zero horizontal stress in the uniaxial case. An increase in the horizontal prestrain modifies the material behaviour, but 
this modification is cancelled out by an increase of the orthogonal vertical prestrain, without obtaining the uniaxial behaviour. The nonlinear behaviour could be explained by fillers aligning in the direction of the applied prestrain, leading to the observed moduli increase.

If this experiment is technically accessible, the interpretation of the measurements required a series of assumptions to estimate the local stress and strain fields. Modifying the experimental set up would limit the amount of approximations needed, for example by adding (i) the measurement of the reaction force in the axis orthogonal to the DMA direction and (ii) the full-field strain measurements in the center of the cross specimen. The drawback is an actual increase of the complexity of the experimental set up. Further experiments are needed in order to estimate precisely the experimental and modelling errors.

\section{Acknowledgements}

The work of AA was financially supported by DGA, Délégation Générale pour l'Armement (France). The authors would like to thank Mrs Amiet (DGA) for supporting this project and $\mathrm{P}$. Pinard for his contribution to the experimental work.

\section{References}

[1] R.A. Schapery, A micromechanical model for non-linear viscoelastic behavior of particle reinforced rubber with distributed damage, Eng. Fract. Mech. 25 (1986) 845.

[2] S. Ozupek, E.B. Becker, Constitutive modeling of high-elongation solid propellants, J. Eng. Mater. Technol. - Trans. ASME 114 (1) (1992) 111.

[3] S. Ozupek, E.B. Becker, Constitutive equations for solid propellants, J. Eng. Mater. Technol. - Trans. ASME 119 (1997) 125.

[4] G. Ravichandran, C.T. Liu, Modeling constitutive behavior of particulate composites undergoing damage, Int. J. Solids Struct. 32 (1995) 979.

[5] G.D. Jung, S.K. Youn, A nonlinear viscoelastic constitutive model of solid propellant, Int. J. Solids Struct. 36 (1999) 3755

[6] C. Nadot-Martin, H. Trumel, A. Dragon, Morphology-based homogenization for viscoelastic particulate composites. Part I: viscoelasticity sole, Eur. J. Mech. A - Solid 22 (1) (2003) 89.

[7] C. Nadot-Martin, A. Dragon, H. Trumel, A. Fanget, Damage modelling framework for viscoelastic particulate composites via a scale transition approach, J. Theor. Appl. Mech. 44 (3) (2006) 553.

[8] C. Nadot-Martin, M. Touboul, A. Dragon, A. Fanget, Direct Scale Transition Approach for Highly-filled Viscohyperelastic Particulate Composites: Computational Study, ISTE/Wiley, 2008 (chapter 12), pp. 218-237.
[9] F. Xu, N. Aravas, P. Sofronis, Constitutive modeling of solid propellant materials with evolving microstructural damage, J. Mech. Phys Solids 56 (2008) 2050

[10] K. Matous, H.M. Inglis, X. Gu, D. Rypl, T.L. Jackson, P.H. Geubelle, Multiscale modeling of solid propellants: from particle packing to failure, Compos. Sci. Technol. 67 (2007) 1694.

[11] P. Mason, The viscoelastic behavior of rubber in extension, J. Appl. Polym. Sci. 1 (1) (1959) 63

[12] C.K.L. Davies, A.G. Thomas, K. Akutagawa, The effect of low molar mass liquids on the dynamic mechanical properties of elastomers under strain, Prog. Rubber Plast. Technol. 12 (3) (1996) 174.

[13] E.A. Meinecke, S. Maksin, Influence of large static deformation on the dynamic properties of polymers. Part II influence of carbon black loading, Rubber Chem. Technol. 54 (1981) 857.

[14] J.L. Sullivan, V.C. Demery, The nonlinear viscoelastic behavior of a carbon black-filled elastomer, J. Polym. Sci. Pol. Phys. Ed. 20 (1982) 2083.

[15] K. Arai, J.D. Ferry, Differential dynamic shear moduli of various carbon-black-filled rubbers subjected to large step shear strains, Rubber Chem. Technol. 59 (1986) 605

[16] A. Voet, J.C. Morawski, Dynamic mechanical and electrical properties of vulcanizates at elongations up to sample rupture, Rubber Chem. Technol. 47 (1974) 765

[17] N.K. Dutta, D.K. Tripathy, Influence of large static deformations on the dynamic mechanical properties of bromobutyl rubber vulcanizates: part I. Effect of carbon black loading, Polym. Test. 9 (1990) 3.

[18] J.J.C. Busfield, C. Deeprasertkul, A.G. Thomas, The effect of liquids on the dynamic properties of carbon black filled natural rubber as a function of pre-strain, Polymer 41 (2000) 9219.

[19] R.L. Warley, D.L. Feke, I. Manas-Zloczower, Transient effects in dynamic modulus measurement of silicone rubber, part 2: effect of mean strains and strain history, J. Appl. Polym. Sci. 104 (2007) 2197.

[20] N. Suphadon, A.G. Thomas, J.J.C. Busfield, The viscoelastic behaviour of rubber under a small simple shear oscillation superimposed on a large pure shear, Polym. Test. 29 (4) (2010) 440.

[21] A. Adicoff, A.H. Lepie, Effect of tensile strain on the use of the WLF equation, J. Appl. Polym. Sci. 14 (1970) 953.

[22] A. Azoug, A. Constantinescu, R.M. Pradeilles-Duval, M.F. Vallat, R. Nevière, B. Haidar, Effect of the sol fraction and hydrostatic deformation on the viscoelastic behavior of prestrained highly filled elastomers, J. Appl. Polym. Sci. 127 (2013) 1772.

[23] N. Suphadon, A.G. Thomas, J.J.C. Busfield, Viscoelastic behavior of rubber under a complex loading, J. Appl. Polym. Sci. 113 (2009) 693.

[24] N. Suphadon, A.G. Thomas, J.J.C. Busfield, The viscoelastic behavior of rubber under a complex loading. II. The effect large strains and the incorporation of carbon black, J. Appl. Polym. Sci. 117 (2010) 1290.

[25] A. Azoug. Micromécanismes et comportement macroscopique d'un élastomère fortement chargé. $\mathrm{PhD}$ Thesis, Ecole Polytechnique, 2010.

[26] A.I. Medalia, Effect of carbon black on dynamic properties of rubber vulcanizates, Rubber Chem. Technol. 51 (1978) 437

[27] A. Thorin, A. Azoug, A. Constantinescu, Influence of prestrain on mechanical properties of highly-filled elastomers: measurements and modeling, Polym. Test. 31 (2012) 978.

[28] K. Matous, M. Kulkarni, P. Geubelle, Multiscale cohesive failure modeling of heterogeneous adhesives, J. Mech. Phys. Solids 56 (2008) 1511.

[29] R. Nevière, An extension of the time-temperature superposition principle to non-linear viscoelastic solids, Int. J. Solids Struct. 43 (2006) 5295 